\title{
Fecal Microbiota Transplantation in Intestinal Disorders: A Primer for Physicians
}

\author{
Arshdeep Singh ${ }^{1}$, Ramit Mahajan ${ }^{2}$, Vandana Midha ${ }^{3}$, Harmeet Kaur ${ }^{4}$, Ajit Sood ${ }^{5}$
}

\begin{abstract}
Gut microbiota has emerged as an important player in the pathogenesis of various intestinal and extraintestinal diseases. Microbiota-targeted therapies, such as probiotics, prebiotics, antibiotics, dietary interventions, and fecal microbiota transplantation (FMT), are therefore being considered and tested as therapies for such diseases. In this review, we discuss the mechanisms and clinical applications of FMT with focus on Clostridioides difficile infection, inflammatory bowel disease, and irritable bowel syndrome. We also discuss practical issues concerning the clinical use of FMT.
\end{abstract}

Keywords: Clostridioides difficile infection, Fecal microbiota transplantation, Inflammatory bowel disease, Irritable bowel syndrome, Ulcerative colitis.

Journal of Gastrointestinal Infections (2020): 10.5005/jp-journals-10068-3041

\section{INTRODUCTION}

Fecal microbiota transplantation (FMT) is the administration of feces, including both structural and functional components of fecal microbial community, from a healthy donor into the gastrointestinal (GI) tract of an individual, with the aim of correcting gut microbial dysbiosis. ${ }^{1}$ The use of human feces as a therapeutic agent dates back to the 4th century AD when Chinese physicians used fecal suspensions for treatment of food poisoning and diarrhea. Ancient Chinese medical literature also describes a "yellow soup" that was used for abdominal diseases with pain abdomen, fever, vomiting, and diarrhea. ${ }^{2}$ Transfaunation-the transfer of fresh feces from healthy animals to diseased animals - was extensively used in the 17th century in veterinary medicine. ${ }^{3}$ The first report of FMT in English literature dates back to 1958 when Eiseman et al. described fecal water enema as a therapy for pseudomembranous colitis. ${ }^{4}$ With increasing antibiotic use, the incidence of recurrent Clostridioides difficile infection ( $\mathrm{rCDI}$ ) has risen in the past decade, and FMT has emerged as an effective therapy for these patients. The efficacy of FMT in rCDI further stimulated the use of FMT in inflammatory bowel disease (IBD) and irritable bowel syndrome (IBS), although the efficacy and safety need to be established. ${ }^{5,6}$ Accumulating shreds of evidence in the last decade point toward a role of microbiota beyond the gut and clinical use of FMT is hence shifting from "infectious" to "noninfectious" disorders"-9 (Fig. 1). This review focuses on the mechanisms and clinical applications of FMT in Gl diseases.

\section{"Normal" Gut Microbiome}

The human Gl tract contains a diverse microbial community comprising more than 100 trillion microorganisms including bacteria, fungi, viruses, and archaea. ${ }^{10}$ Although the entire spectrum of microbial community plays an integral role in shaping $\mathrm{Gl}$ physiology, bacteria are the most extensively studied because of their abundance and relative ease of phylogenetic identification and quantification. The gut bacterial genome consists of more than 5 million genes as compared to 25,000 genes of humans, making it a much larger and vital component of the human bioecosystem. ${ }^{11}$
1,2,5 Department of Gastroenterology, Dayanand Medical College and Hospital, Ludhiana, Punjab, India

${ }^{3}$ Department of Medicine, Dayanand Medical College and Hospital, Ludhiana, Punjab, India

${ }^{4}$ Department of Obstetrics and Gynecology, Dayanand Medical College and Hospital, Ludhiana, Punjab, India

Corresponding Author: Arshdeep Singh, Department of Gastroenterology, Dayanand Medical College and Hospital, Ludhiana, Punjab, India, e-mail: drarshdeepsingh@gmail.com

How to cite this article: Singh A, Mahajan R, Midha V, et al. Fecal Microbiota Transplantation in Intestinal Disorders: A Primer for Physicians. J Gastrointest Infect 2020;10(1):16-25.

Source of support: Nil

Conflict of interest: None

Various Gl, hepatobiliary, metabolic, neoplastic, and neurological disorders have been linked to changes within the bacteriome. Nonetheless, there appears a strong interdependence between bacteriome, virome, and fungome; any imbalance can impact the state of health. ${ }^{12}$

The colon is one of the most densely populated microbial habitats with approximately $10^{12}$ bacterial cells per milliliter. The dominant microbial phyla are Firmicutes, Bacteroidetes, Actinobacteria, Proteobacteria, Fusobacteria, and Verrucomicrobia. Of these, Firmicutes (comprising Lactobacillus, Bacillus, Clostridioides, Clostridium, Eubacterium, Enterococcus, and Ruminococcus) and Bacteroidetes (consisting of Bacteroides and Prevotella) represent $>90 \%$ of gut microbiota. ${ }^{13}$ It is important to recognize that bacterial composition within an individual is variable and dynamic, and therefore, defining a "normal" healthy gut microbiome is complicated. The microbial makeup is determined by host genetics, anatomical location within the Gl tract, age, and environmental factors like diet and use of antibiotics and proton pump inhibitors. Also, there are interindividual variations driven by enterotypes and external influences like geographical locations, province, ethnicity, lifestyle, and dietary and cultural habits ${ }^{14}$ (Fig. 2). 


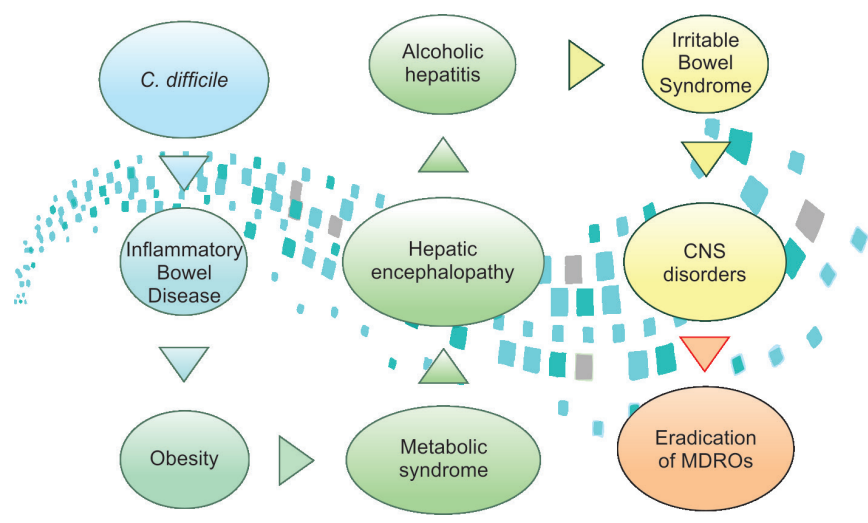

Fig. 1: Expanding indications of fecal microbiota transplantation

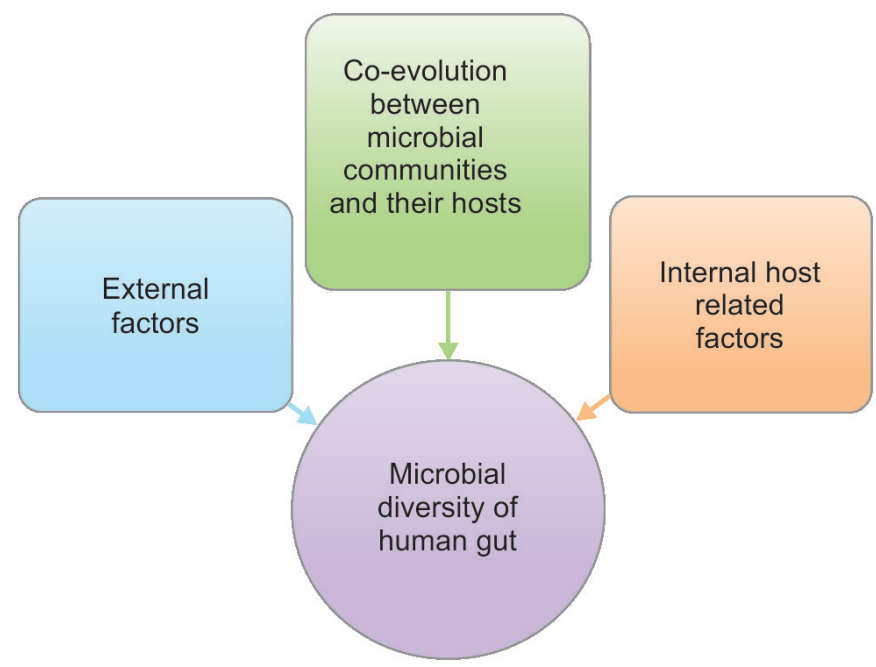

Fig. 2: Factors influencing the composition and diversity of gut microbiome

When there are factors that change the gut microbiome, physiological adaptation attempts to maintain its natural environment, indicating resilience. Additionally, immunocompetent hosts, although constantly exposed to intestinal pathogens, do not develop the disease because there is resistance to colonization of pathogens due to direct interactions between microbes as well as indirect mechanisms mediated by the mucosal immune system. ${ }^{15}$ However, disruption of the harmony of a healthy intestinal milieu may lead to lasting changes in the gut microbial constitution (i.e., dysbiosis) that may be demanding as well as disruptive (leading to a diseased state) for a complex and interconnected system like the Gl tract.

\section{Rationale and Mechanisms for Fecal Microbiota Transplantation}

The idea that normalization of an altered gut microbiome and restoration of the microbial community alleviates disease is the backbone of the science of FMT. However, what works in an FMT and what changes are brought about by FMT remain ambiguous. Microbial therapeutic science is still in its infancy. The use of whole stool preparation for FMT has obviated the need to analyze the active moiety in the microbial ecosystem, be it bacteria, fungi, viruses, metabolites, or an interaction between these components. Herein, we explore some of the potential mechanisms of FMT.
Fecal Microbiota Transplantation-associated Changes in Gut Microbial Structure and Composition

Loss of bacterial diversity and alterations in the relative distribution of microorganisms may lead to persistence and proliferation of pathobionts, evasion of host inflammatory response, and development of disease. ${ }^{16}$ For example, dysbiosis characterized by complete disappearance of Bacteroidetes, reduction in Firmicutes, and increase in the relative abundance of Proteobacteria is noted in patients with $C$. difficile infection (CDI). ${ }^{17}$ Rapid restoration of the microbial community has been noted as early as 24 hours after FMT, resulting in therapeutic benefit. ${ }^{18}$ The diegesis, however, in IBD where dysbiosis is not as conspicuous, is not yet clear. Dysbiosis in inflammatory bowel disease is characterized by reduced diversity within Firmicutes, reduction/spatial reorganization of Bacteroidetes, and an increased proportion of Proteobacteria. ${ }^{19}$ FMT, as in CDI, is expected to correct these abnormalities and reduce gut inflammation. However, efforts in this direction have not yielded consistent results and no specific group of microbes have been established in relation to response to FMT in ulcerative colitis (UC). ${ }^{20-23}$ The microbial composition in IBS (both diarrheapredominant and constipation-predominant subgroups) is specified by lower Lactobacillus and Bifidobacterium and higher Escherichia coli and Enterobacter. ${ }^{24}$ Akin to IBD, however, various studies using FMT as therapy for IBS have not been able to produce uniform and reproducible results.

This is not unexpected as the pathophysiological mechanisms in IBD and IBS are chronic and multifactorial, involving host genetics and a complex web of environmental influences. On the contrary, $\mathrm{CDI}$ is predominantly an acute microbial disease. Hence, additional mechanisms, apart from restoration of microbial structure, seem to be at play, which determines the outcomes with FMT. The uncertainty surrounding dysbiosis being a cause or an effect of altered immune and metabolic environment further complicates the feasibility of an in-depth analysis of changes brought by FMT.

\section{Competitive Niche Exclusion}

The microorganisms within the GI tract also intensely compete with each other for nutrients and space. For example, sialic acid released by resident Bacteroides species is a nutrient source for $C$. difficile as it contains a sialic acid catabolic operon. ${ }^{25}$ Intestinal microorganisms also release monomeric glucose and $\mathrm{N}$-acetylglucosamine that are used by cohabitants as energy sources. ${ }^{26}$ Similarly, siderophore (iron scavenging molecules)-mediated competition for iron acquisition is seen between human opportunistic pathogens Staphylococcus aureus and Pseudomonas aeruginosa. ${ }^{27}$

C. difficile can grow merely on carbon dioxide and hydrogen gases as sole sources of carbon and energy, respectively. ${ }^{28}$ This gives an edge to $C$. difficile in narrowly differentiated nutritional niches as other microorganisms need a wealth of metabolic functionality for survival. The exclusion of competitive niche by FMT-induced restoration of microbial communities, thereby preventing colonization with pathobionts, is one of the plausible mechanisms of FMT.

\section{Alteration of Secondary Bile Acid Metabolism}

Alteration of secondary bile acid metabolism is particularly important in CDI. The growth and reproductive response of C. difficile depend on the concentration and proportion of primary and secondary bile acids in the intestinal lumen. Secondary bile acids, normally the dominant bile acids in colonic lumen and 
inhibitors of germination of $C$. difficile spores, are deficient in $\mathrm{rCDI}$. FMT results in restoration of normal colonic microbial ecology (rehabilitation of Lachnospiraceae, Ruminococcaceae families) that reinstates normal bile acid composition and normalizes bile acid metabolism in the colon, producing an unfavorable environment for C. difficile to survive. ${ }^{29,30}$

\section{Immune-mediated Mechanisms}

The mucosal immune system is characterized by its exclusivity. Commensal microorganisms are indispensable for development as well as maturation of mucosal immunity. Several immune mechanisms work in tandem with the microbiota to establish and maintain gut homeostasis. Secretion of mucin glycoproteins by goblet cells, IgA by plasma cells, and antimicrobial peptides by epithelial cells limits the colonization of pathogens. ${ }^{31}$ Toll-like receptors, present on the intestinal epithelial and lymphoid cells recognize microbe-associated molecular patterns of the commensals and via regulatory $T$ (Treg)-cell differentiation, retinoic acid, and aryl hydrocarbon receptor signaling, contain inflammatory responses. Gut microbiota plays an important role in modulating and effecting the differentiation of T cells into Th1, Th2, and Th17 or into Treg cells. ${ }^{32}$ Commensal microbiome-derived metabolites often have antimicrobial properties that resist colonization by noncommensal pathogenic species. ${ }^{33}$ Therefore, a dysbiotic microbial community, at a time when maturation of the innate immune system takes place, has a potential to elicit inapt immune responses (hyperactivation of Th1 and Th17 cells) and lead to the loss of immunological tolerance. Uncontrolled colonic inflammation leads to oxidative stress through the generation of reactive oxygen species that is likely to promote the disproportionate growth of aerotolerant anaerobes, such as Proteobacteria and Actinobacteria, resulting in decreased bacterial diversity and vicious cycle ensues. ${ }^{34}$ FMT has been postulated to correct dysbiosis and control inflammation that might be driven by gut microbiota. Studies in mouse models have demonstrated immunomodulatory effects of FMT by restoring the balance of enteric immune system. ${ }^{35-37}$ However, the only randomized trial that looked into the immunomodulatory effects of FMT did not find significant changes in T-cell subsets. ${ }^{23}$

\section{Clinical Applications of Fecal Microbiota Transplantation}

\section{C. difficile Infection}

Since the pioneering work of Eiseman, Chief of Surgery at Denver General Hospital, in the late 1950s, multiple controlled trials and cohort studies have demonstrated that FMT is a highly effective therapeutic strategy in patients suffering from $\mathrm{rCDI}^{4,38-41} \mathrm{~A}$ recent systematic review and meta-analysis including thirty-seven studies [seven randomized controlled trials (RCTs) and 30 case series] reported that FMT was more effective than vancomycin in treating $\mathrm{rCDI}$. Clinical resolution across all studies was $92 \% .{ }^{42} \mathrm{~A}$ more recent updated metaanalysis (8 RCTs involving 537 patients with rCDI) demonstrated a significantly lower recurrence rate of diarrhea with $\mathrm{FMT}^{43}$ In another RCT of patients with rCDI, FMT, and vancomycin when used in combination were superior to fidaxomicin or vancomycin alone. $^{44}$ The majority of the published literature on FMT in rCDI employs a single session of FMT. However, in patients with severe $\mathrm{CDI}$, a single intervention is unlikely to achieve complete resolution. Sequential FMTs in rapid cycles with continuation of vancomycin or pseudomembrane-driven sequential FMT (selective use of vancomycin until complete resolution of pseudomembranes followed by FMT) has been proposed for these patients. ${ }^{45-47}$ Early FMT (as a first-line treatment) has also been shown to reduce mortality in severe $\mathrm{CDI} .{ }^{48}$ However, more data are needed before FMT is recommended as a treatment for the first episode of CDI. ${ }^{49,50}$ A European consensus on FMT in clinical practice recommends FMT as a treatment option for both mild and severe recurrent and refractory CDI. ${ }^{51}$

\section{Inflammatory Bowel Disease}

FMT was attempted in UC in 1989 by Bennet and Borody. ${ }^{52,53}$ However, interest in FMT for IBD was heightened after two RCTs evaluating FMT for induction of remission were published with conflicting results in $2015 .^{20,22}$ Since then, there have been many cohort studies $^{54-56}$ and two more RCTs-both from Australiademonstrating efficacy of FMT in inducing remission in UC. ${ }^{21,23}$ Our group also evaluated the role of multisession colonoscopic FMT in steroid-dependent UC. Steroid-free clinical remission was achieved in $46.3 \%$ patients, whereas clinical response and endoscopic remission were achieved in $75.6 \%$ and $63.4 \%$ patients, respectively, at 22 weeks and all (100\%) patients with clinical response were able to withdraw steroids. ${ }^{57}$ The Cochrane review on FMT in IBD concluded that although FMT demonstrated benefit in inducing remission in UC, no solid conclusions can be drawn due to small study sizes and low quality of evidence. Additionally, there was uncertainty about the rate of serious adverse events. ${ }^{6}$

Data concerning durability of response and long-term outcomes after FMT in UC are scarce. In one of the first published RCTs, most of the patients who achieved remission were unable to sustain and relapsed on follow-up. ${ }^{58}$ Our group, in a randomized pilot study, evaluated 8 weekly colonoscopic infusion of FMT for maintaining remission in UC. Patients were randomized to receive either FMT or placebo in addition to stable doses of 5-aminosalicylates plus azathioprine. Among participants allocated to pharmacotherapy plus FMT group, 27 of 31 (87.1\%) were able to maintain steroid-free clinical remission at week 48, vs 20 of 30 (66.7\%) patients assigned to the pharmacotherapy plus placebo group $(p=0.111)$. Endoscopic [FMT: $18 / 31$ (58.1\%) vs placebo: $8 / 30(26.7 \%), p=0.026$ ] and histological [FMT: $14 / 31$ (45.2\%) vs placebo: $5 / 30(16.7 \%), p=0.033$ ] remissions were maintained in a significantly higher number of patients receiving FMT in addition to pharmacotherapy ${ }^{59}$ Another study from China has also reported long-term clinical benefits achieved from FMT with maintenance therapy. ${ }^{60}$ Larger studies are, however, needed to corroborate these findings.

There are limited studies with variable and heterogeneous protocols that suggest a potential role of FMT in Crohn's disease. ${ }^{61-64}$ However, no definitive inferences can be made at the moment and more data from larger RCTs are needed.

\section{Irritable Bowel Syndrome}

An altered gut microbiome has been identified in IBS patients, and FMT has been suggested to treat IBS. ${ }^{65-67}$ In one of the earliest studies of FMT in IBS, $36 \%$ of the patients could achieve complete resolution of symptoms and $10.9 \%$ achieved partial relief. ${ }^{53}$ This study, however, included patients with IBD and CDI. Subsequently, randomized trials demonstrated encouraging results with FMT in constipation predominant, diarrhea-predominant, and mixed IBS. ${ }^{68-72}$ A recent meta-analysis of 5 RCTs involving 267 participants however found that the relative risk of IBS symptoms not improving after FMT vs placebo was 0.98 (95\% Cl 0.58-1.66), although there was significant heterogeneity detected between studies $\left(I^{2}=78 \%, p=0.001\right)$. On subgroup analysis fresh or frozen donor stool delivered via colonoscopy was beneficial but no benefit 
was observed when delivery was via the upper Gl tract. ${ }^{73}$ Another meta-analysis, concluded that FMT is not superior to placebo in the treatment of IBS. ${ }^{74}$ Heterogeneity in the protocols for performing FMT including route and frequency of administration, donor factors, duration of follow-up, and IBS subtypes affects the outcomes of currently available trials. Further studies with uniformity in protocols and homogeneous patient populations are needed.

\section{Essentials for Performing Fecal Microbiota Transplantation}

Preprocedure

Informed consent: FMT is an investigational procedure, regulated by the United States Food and Drug Administration under investigational new drug. At the moment, the clinical use of FMT beyond clinical trials is not recommended except for CDI. The anticipated benefits/risks of FMT, alternative therapies, and potential complications during recuperation and the likelihood of achieving therapeutic goals should be discussed with the patient in detail before an informed decision about undergoing FMT is made.

Donor selection: Donor selection is the most important aspect of FMT. It is imperative to select a donor who is healthy (free of any disease or infirmity) and does not have had exposure to antibiotics or infectious illnesses within three months of stool donation. The assessment of medical history and risk assessment of potential donors is usually done by a screening questionnaire. Once a potential donor is identified, a detailed physical and laboratory evaluation is carried out (Table 1). The principle behind rigorous donor selection protocols is to reduce and prevent any adverse event related to the infused fecal material. ${ }^{51}$ It is also important to maintain a close contact and follow-up of the donor to identify problems related to donation and also track the medical and dietary history of the donor. There is evidence to suggest that animal-based diets and diets rich in fats (rich in omega-6 polyunsaturated fats), proteins, refined carbohydrates, and food additives/emulsifiers negatively impact the gut microbiota and therefore may be a deterrent to stool donation. ${ }^{75}$ Regular (at least every three months) fecal and biochemical testing (for infections and other comorbidities) is also necessary and FMT products should be used only if the donor is found acceptable in the repeat screen as well as on the day of donation.

Clinical responses to FMT are variable: even for the recipients who are subjected to the same donor and same FMT protocols. The genetic background, diet, and microbial and metabolic profiling of both the recipient and the donor determine the overall outcome of FMT. Thus "one stool fits all" approach does not hold true in the context of treating microbial dysbiosis-associated disorders. It is therefore important to understand that selection of donors based solely on clinical screening guidelines provides no guarantee of FMT success, although it reduces the chances of an adverse event. In the future, apart from screening for infectious agents, analysis of microbiome diversity indices, screening for nonbacterial members of gut microbiota, screening for antibiotic resistance as well as understanding and interpreting the screening results taking into consideration the reference population will hold the key to selecting donors in a righteous way. Also, analyzing the diversity in keystone populations may enable selecting specific donors for specific FMT indications. $^{76}$

Preparation of slurry: The procedure of performing FMT has evolved over centuries from consumption of fresh, dry, or fermented stool mixed in water to the preparation of fecal slurry. Much of the developments in FMT, including purification of microbiota by filtration or centrifugation, cryopreservation, and development of lyophilized FMT capsules, have occurred in the last decade. ${ }^{77}$ The refined techniques minimize manual handling of the fecal sample and are a step toward standardization of the laboratory processes. Employing laminar flow cabinet or tissue culture hood with ultraviolet- $C$ germicidal lamp during the preparation of the fecal slurry can prevent contamination of fecal samples, although experience with its use is primal.

Fresh vs frozen donor fecal sample: FMT infusions prepared from fresh or frozen $\left(-80^{\circ} \mathrm{C}\right)$ stool have demonstrated comparable efficacy, although a decline in microbial viability has been noted in the frozen stool. ${ }^{78,79}$ In FMT preparations that are frozen for 9-12 months, there is an observed decline in relative abundances of Bifidobacterium and Lactobacillus, and these changes may be responsible for the loss of efficacy in $\mathrm{rCDI}$ in rodents. ${ }^{80}$

A recent $\mathrm{RCT}$ estimated the comparative $\mathrm{rCDI}$ remission rates of 100 and $83 \%$ with the use of fresh and frozen preparations, respectively, and $78 \%$ for lyophilized preparations. ${ }^{81}$ Although the data are conflicting, majority of the experts believe that outcomes do not differ between fresh and frozen stool. Hence, many centers across the world use fecal slurries stored at $-80^{\circ} \mathrm{C}$. Banking material is thawed to room temperature and then used for FMT. Refreezing, once the fecal sample has been thawed, is not carried out. Maximum shelf life of frozen slurry, recommended by most experts, is 6 months. ${ }^{82}$

The authors opine that fresh FMT may be superior to frozen FMT and therefore practice delivering fecal slurry within 6 hours of collection of stool and 1 hour of preparation of the slurry. This, however, needs corroboration in larger well-designed trials. Till more evidence is available, both frozen and fresh fecal samples can be used, expecting similar clinical efficacy and safety.

Aerobic vs anaerobic preparation: Many bacterial populations in the human gut are obligate or facultative anaerobes. Preparing FMT slurry in an anaerobic environment may preserve these anaerobes. There is evidence that the proportion of viable bacteria reduces when donor stool sample is processed in ambient air as compared to immediate anaerobic processing. ${ }^{79}$ Although quality head-to-head comparisons of aerobic and anaerobic preparations are lacking, both anaerobically and aerobically prepared FMTs have appeared efficient. ${ }^{20,21,23,78}$

Use of antibiotics: The use of antibiotics prior to FMT is debatable although a larger school of thought is that it may help in alteration of gut microbiota in recipients and aid in increasing the efficacy of FMT by restoring the ecological niches. Mice studies and a metaanalysis of human studies suggest that antibiotic pretreatment before FMT may be beneficial. ${ }^{83,84}$ More studies are, however, needed to determine the effects on bacterial strain engraftment, clinical efficacy, and cost-effectiveness.

\section{Procedure}

Route of administration: The current routes of administration of fecal slurry are via endoscopy (colonoscopy/gastroduodenoscopy), nasoduodenal/nasojejunal or colonic tubes, enema, and via encapsulation.

For colonoscopic route of administration, bowel preparation is done using polyethylene glycol-based solutions similar to bowel preparation for diagnostic colonoscopy. Some FMT centers recommend 4 to $8 \mathrm{mg}$ of oral loperamide preprocedure to aid the retention of the transplant material. Delivery via colonoscopy 
Table 1: Donor screening and selection criteria

\begin{tabular}{|c|c|}
\hline \multicolumn{2}{|c|}{ Essential components of donor screening questionnaire } \\
\hline Infectious diseases & $\begin{array}{l}\text { - History or exposure to infectious diseases with chronic activity (HIV, HBV, HCV, syphilis, tuberculosis, Chagas } \\
\text { disease, strongyloidiasis) } \\
\text { - } \text { Vaccination with a live attenuated vaccine within the last } 6 \text { months } \\
\text { - } \quad \text { Any currently active infections or those treated within the last } 6 \text { months }\end{array}$ \\
\hline At-risk behavior & $\begin{array}{l}\text { - Use of illegal drugs and/or consumption of alcohol/tobacco } \\
\text { - } \quad \text { Ongoing high-risk sexual behavior } \\
\text { - } \quad \text { purrent occupation in a setting facilitating acquisition of potential pathogens (e.g., healthcare professionals with } \\
\text { - Tattoo, piercing, or acupuncture within the last } 6 \text { months } \\
\text { - } \quad \text { Previous tissue/organ transplantation } \\
\text { - Blood transfusion within the last } 6 \text { months }\end{array}$ \\
\hline Medical history & $\begin{array}{l}\text { - } \quad \text { Any chronic disease } \\
\text { - } \quad \text { Previous oncological or immunological diagnosis, even if not active at the time of screening } \\
\text { - } \quad \text { Antibiotic treatments or chemotherapy ongoing, scheduled, or received within the last } 3 \text { months }\end{array}$ \\
\hline $\begin{array}{l}\text { Current signs and } \\
\text { symptoms }\end{array}$ & $\begin{array}{l}\text { - } \quad \text { Diarrhea, hematochezia, or any other gastrointestinal symptom within the last } 3 \text { months } \\
\text { - } \quad \text { Fever or rash within the last } 3 \text { months } \\
\text { - } \quad \text { Any other clinical sign or symptom within the last } 3 \text { months }\end{array}$ \\
\hline \multicolumn{2}{|l|}{ Stool analysis } \\
\hline $\begin{array}{l}\text { Bacterial enteric } \\
\text { pathogens }\end{array}$ & $\begin{array}{ll}\text { - } & \text { Enteroaggregative E. coli (EAEC) } \\
\text { - } & \text { Enteropathogenic E. coli (EPEC) } \\
\text { - } & \text { Enterotoxigenic E. coli (ETEC) } \\
\text { - } & \text { Enteroinvasive E. coli (EIEC) } \\
\text { - } & \text { Shiga-like toxin-producing E. coli (STEC) } \\
\text { - } & \text { Shigella spp. } \\
\text { - } & \text { Campylobacter spp. } \\
\text { - } & \text { Salmonella spp. } \\
\text { - } & \text { Yersinia spp. } \\
\text { - } & \text { Vibrio spp. } \\
\text { - } & \text { Clostridioides difficile } \\
\text { - } & \text { Helicobacter pylori (Fecal antigen) }\end{array}$ \\
\hline $\begin{array}{l}\text { Multidrug-resistant } \\
\text { organisms }\end{array}$ & $\begin{array}{ll}\text { - } & \text { Extended-spectrum beta-lactamase-producing bacteria (ESBLs) } \\
\text { - } & \text { Vancomycin-resistant enterococci (VRE) } \\
\text { - } & \text { Carbapenem-resistant bacteria (CRE) } \\
\text { - } & \text { Methicillin-resistant Staphylococcus aureus (MRSA) }\end{array}$ \\
\hline Viruses & $\begin{array}{ll}\text { - } & \text { Norovirus } \\
\text { - } & \text { Rotavirus } \\
\text { - } & \text { Adenovirus } \\
\text { - } & \text { Astrovirus } \\
\text { - } & \text { Sapovirus } \\
\text { - } & \text { SARS-CoV-2 }\end{array}$ \\
\hline Parasites & $\begin{array}{ll}\text { - } & \text { Cryptosporidium spp. (PCR or antigen) } \\
\text { - } & \text { Giardia lamblia (PCR or antigen) } \\
\text { - } & \text { Isospora and Microspora (PCR or microscopy) }\end{array}$ \\
\hline Others & Fecal occult blood testing \\
\hline \multicolumn{2}{|l|}{ Blood analysis } \\
\hline General & $\begin{array}{ll}\cdot & \text { Erythrocyte sedimentation rate (ESR) } \\
\cdot & \text { C-reactive protein (CRP) } \\
\cdot & \text { Serum creatinine } \\
\text { - } & \text { Serum sodium, potassium } \\
\text { - } & \text { Aspartate aminotransferase (AST) } \\
\text { - } & \text { Alanine aminotransferase (ALT) } \\
\text { - } & \text { Alkaline phosphatase (ALP) } \\
\text { - } & \text { Gamma-glutamyl transferase (GGT) } \\
\text { - } & \text { Bilirubin } \\
\text { - } & \text { Albumin } \\
\text { - } & \text { Total blood cell count } \\
\cdot & \text { Fasting glucose } \\
\text { - } & \text { Lipid panel } \\
\end{array}$ \\
\hline
\end{tabular}




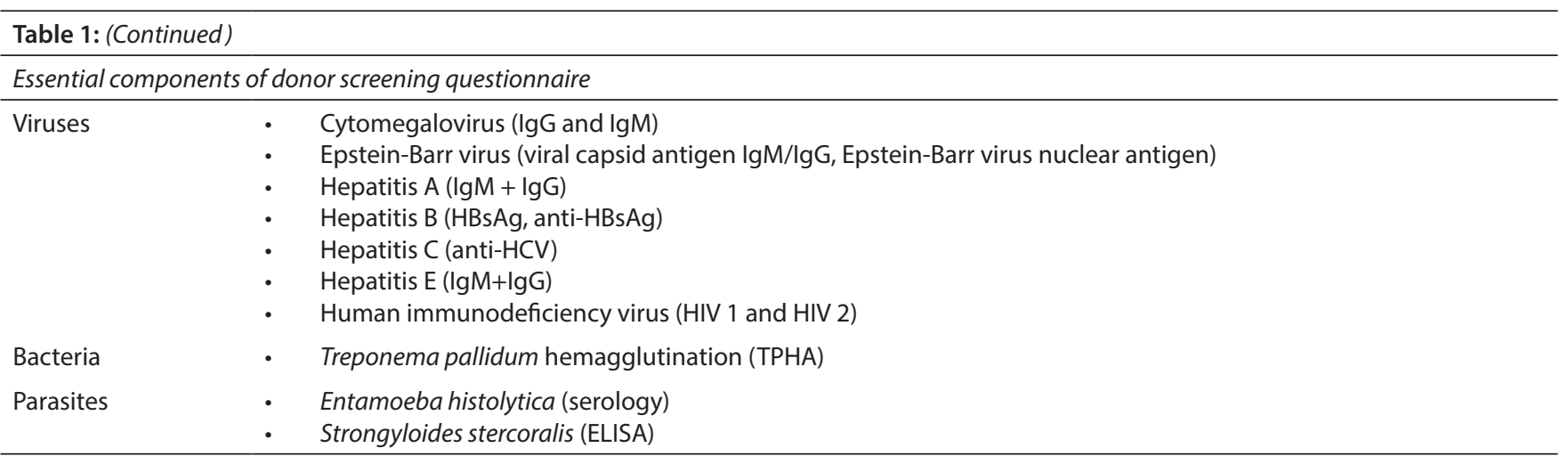

is superior for slurry retention as compared to enemas and also for delivering larger quantity of fecal slurry. Enemas are contrastingly less invasive and allow frequent interventions. However, performing frequent enemas on a domiciliary basis is a cumbersome procedure that requires intensive patient training and adequate fecal slurry storage facilities. FMT performed via colonoscopy also carries the risks associated with colonoscopic procedure, including bowel perforation. Colonic transendoscopic enteral tubing (TET) placed in midgut or colon has been described for patients who need frequent/maintenance FMTs. ${ }^{85}$ However, clipping the tube on a diseased mucosa and risk of anal incontinence associated with colonic TET may hinder its widespread use.

For upper GI delivery, gastric acidic milieu has the propensity to damage the fecal slurry (although researchers have attempted to negate this by using proton pump inhibitors or delivering the fecal slurry into duodenum or jejunum) and has poor aesthetics and a higher incidence of adverse events. ${ }^{86,87}$ The invasiveness and discomfort of a nasal tube makes nasoenteric administration the least attractive modality of all.

The efficacy and safety of oral FMT capsules have been demonstrated for rCDI as well as IBD, although not in IBS. ${ }^{74,88,89}$ These are likely to be favored by patients over endoscopic options for their ease and convenience. Dosage, frequency of administration, total treatment duration, and long-term response with oral capsules, however, needs to be studied.

There is no consensus on the most appropriate route of administration of FMT and more data are needed before recommendations are made.

Dosage and frequency of administration: The dose and the frequency of FMT also vary across the literature. It is difficult to standardize the amount of stool required for FMT with respect to microbial diversity. Most studies, however, use approximately $50 \mathrm{~g}$ of donor stool.

A single session of FMT has been shown to be efficacious in patients with CDI. ${ }^{38-42}$ However, for severe and fulminant CDI, single FMT is unlikely to achieve sustained benefit and sequential FMTs have resulted in cure rates of $>90 \%$ and decline in mortality from 10.2 to $4.5 \%$ in patients with severe infection and from 43.2 to $12.1 \%$ in fulminant infection. ${ }^{45,46}$ Similarly for UC, which involves complex pathologic mechanisms including immunologic, microbial dysbiosis and genetic factors, therapeutic microbial manipulation with a single session of FMT is unlikely to have sustained benefits. ${ }^{90}$ The reported frequency of administration of FMT in UC varies from limited dosing schedules of 1-2 doses to extremely intense schedule of 41 doses. ${ }^{20-23}$ In published trials on IBS, the frequency of intervention varies from once via colonoscopy to 25 oral capsules daily with variable results. The optimal interval between two sessions of FMT is speculative. Bacterial engraftment in patients with $\mathrm{rCDI}$ has been demonstrated to increase from days 2 to 6 after FMT that achieves a plateau by days 28-45. This engraftment is sustained for months. ${ }^{91}$ However, in IBD and IBS, repeated FMTs are needed for maintaining the efficacy of previous FMT. Although there is no consensus, intervals ranging from 1 to 12 weeks have been described. ${ }^{20-23}$

\section{Postprocedure Monitoring and Safety}

Apart from efficacy, tolerability and safety are equally vital for FMT to gain acceptance in the therapeutic armamentarium of intestinal diseases. The long-term safety of FMT in rCDI has been studied in both immune-competent and immune-compromised patients, and FMT has been found to be a durable, safe, and acceptable treatment option. ${ }^{92-94}$ Adverse events associated with FMT are commonly mild. A systematic review revealed the adverse event incidence rate of $28.5 \%$. The commonest FMT-attributable adverse event was abdominal discomfort. Serious adverse events included infections, relapse of IBD, C. difficile related infection, and death. ${ }^{87}$ The authors have also reported abdominal discomfort, flatulence, abdominal distension, borborygmi, and low-grade fever as the common postprocedural short-term adverse events for FMT in active UC. Long-term adverse events including new-onset urticaria, arthritis/arthralgia, depression, partial sensorineural hearing loss, and allergic bronchitis have also been observed..$^{95}$ In a systematic review and meta-analysis on FMT in IBS, two serious adverse events were reported. One serious adverse event of transient vertigo and nausea developed after the FMT procedure, requiring a few hours of observation in the hospital. The other serious adverse event of suicide in the month following FMT occurred in the placebo group. No other serious adverse events were reported. ${ }^{74}$

More recently, however, extended-spectrum beta-lactamaseproducing $E$. coli bacteremia has been reported in two patients after FMT, highlighting the need of strict donor screening protocols, appropriate patient selection, and a vigilant postprocedure observation. ${ }^{96}$ Intensive postprocedure monitoring for adverse events (defined as events that prevented completion of the procedure and/or resulted in admission to hospital, prolongation of existing hospital stay, another procedure, or subsequent medical consultation) is recommended. Adverse events can be timed as intraprocedural, immediate postprocedural (up to 48 hours), 


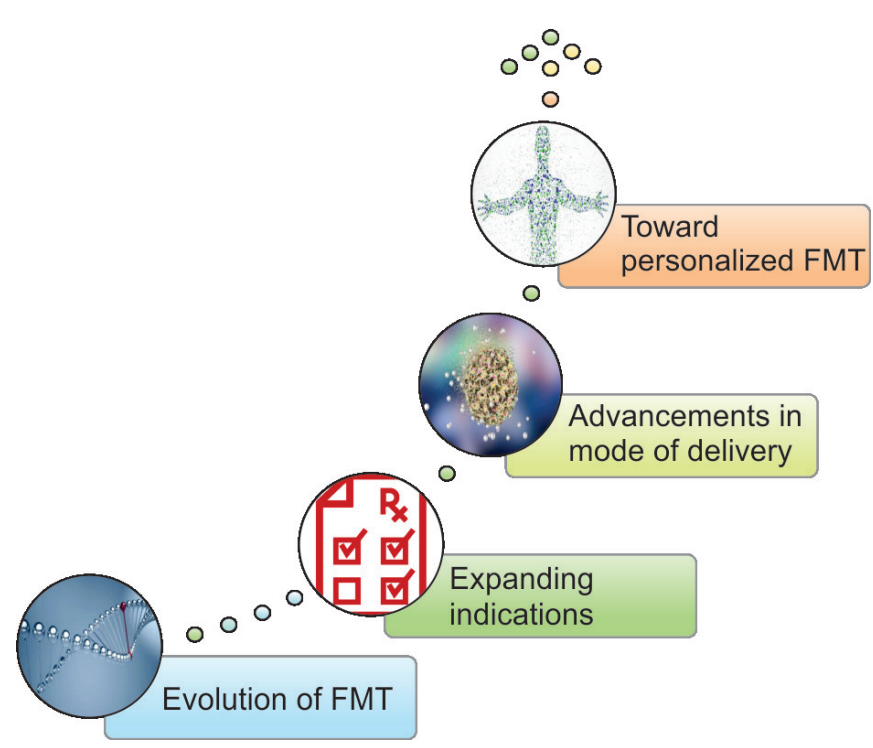

Fig. 3: Fecal microbiota transplantation: the way forward. FMT, fecal microbiota transplantation

postprocedural (up to 14 days), and late (>14 days after the procedure). The authors use a predesigned template to note the adverse events that are graded for their severity as per the American Society of Gastrointestinal Endoscopy report. ${ }^{97}$

\section{The Future}

FMT has emerged as a novel therapy targeting the microbial dysbiosis in various intestinal and extraintestinal diseases. By targeting gut microbial dysbiosis, it also tends to modulate various host physiological responses. Although our understanding of microbiome is expanding, the science of gut microbiota and its clinical implications is still in its early years. The exact mechanism of action of FMT is not known and comprehensive studies incorporating genetic, metagenomic, metatranscriptomic, and metabolomic components are needed to shed more light on the functional ecologies. Microbiota-targeted therapies hold out hopes for potential applications in CDI, IBD, and IBS and also for extraintestinal diseases like metabolic syndrome, alcoholic hepatitis, and neurological and psychiatric illnesses. The optimal mode and route of delivery, usage of cocktail of select microbes and metabolites instead of whole stool, patient selection, effect of concomitant therapies, and impact of diet on outcomes with FMT are some of the many unanswered questions. However, the future seems promising (Fig. 3).

\section{References}

1. Smits LP, Bouter KE, de Vos WM, et al. Therapeutic potential of fecal microbiota transplantation. Gastroenterology. 2013;145(5):946-953. DOI: 10.1053/j.gastro.2013.08.058.

2. Zhang F, Luo W, Shi Y, et al. Should we standardize the 1,700-yearold fecal microbiota transplantation? Am J Gastroenterol 2012;107(11):1755. DOI: 10.1038/ajg.2012.251.

3. DePeters EJ, George LW. Rumen transfaunation. Immunol Lett 2014;162(2 Pt A):69-76. DOI: 10.1016/j.imlet.2014.05.009.

4. Eiseman B, Silen W, Bascom GS, et al. Fecal enema as an adjunct in the treatment of pseudomembranous enterocolitis. Surgery 1958;44(5):854-859.
5. Halkjær SI, Boolsen AW, Günther S, et al. Can fecal microbiota transplantation cure irritable bowel syndrome? World J Gastroenterol 2017;23(22):4112-4120. DOI: 10.3748/wjg.v23.i22.4112.

6. Imdad A, Nicholson MR, Tanner-Smith EE, et al. Fecal transplantation for treatment of inflammatory bowel disease. Cochrane Database Syst Rev 2018;11(11):CD012774.DOI: 10.1002/14651858.CD012774.pub2.

7. Vrieze A, Van Nood E, Holleman F, et al. Transfer of intestinal microbiota from lean donors increases insulin sensitivity in individuals with metabolic syndrome. Gastroenterology 2012;143(4):913-916.e7. DOI: 10.1053/j.gastro.2012.06.031.

8. Bajaj JS, Kassam Z, Fagan A, et al. Fecal microbiota transplant from a rational stool donor improves hepatic encephalopathy: a randomized clinical trial. Hepatology 2017;66(6):1727-1738. DOI: 10.1002/hep.29306.

9. Leshem A, Horesh N, Elinav E. Fecal Microbial Transplantation and Its Potential Application in Cardiometabolic Syndrome. Front Immunol 2019;10:1341. DOI: 10.3389/fimmu.2019.01341.

10. Thursby $E$, Juge N. Introduction to the human gut microbiota. Biochem J 2017;474(11):1823-1836. DOI: 10.1042/BCJ20160510.

11. Zou Y, Xue W, Luo G, et al. 1,520 reference genomes from cultivated human gut bacteria enable functional microbiome analyses. Nat Biotechnol 2019;37(2):179-185. DOI: 10.1038/s41587-018-0008-8.

12. Filyk HA, Osborne LC. The multibiome: the intestinal ecosystem's influence on immune homeostasis, health, and disease. EBioMedicine 2016;13:46-54. DOI: 10.1016/j.ebiom.2016.10.007.

13. Arumugam $M$, Raes J, Pelletier $E$, et al. Enterotypes of the human gut microbiome. Nature 2011;473(7346):174-180. DOI: 10.1038/ nature09944.

14. Rinninella $E$, Raoul $P$, Cintoni $M$, et al. What is the healthy gut microbiota composition? A changing ecosystem across age, environment, diet, and diseases. Microorganisms 2019;7(1):14. DOI: 10.3390/microorganisms7010014.

15. Lawley TD, Walker AW. Intestinal colonization resistance. Immunology 2013;138(1):1-11. DOI: 10.1111/j.1365-2567.2012.03616.x.

16. Thakur $A$, Mikkelsen $H$, Jungersen $G$. Intracellular pathogens: host immunity and microbial persistence strategies. J Immunol Res 2019;2019:1356540. DOI: 10.1155/2019/1356540.

17. Antharam VC, Li EC, Ishmael A, et al. Intestinal dysbiosis and depletion of butyrogenic bacteria in Clostridium difficile infection and nosocomial diarrhea. J Clin Microbiol 2013;51(9):2884-2892. DOI: 10.1128/JCM.00845-13.

18. Weingarden A, González A, Vázquez-Baeza Y, et al. Dynamic changes in short- and long-term bacterial composition following fecal microbiota transplantation for recurrent Clostridium difficile infection. Microbiome 2015;3:10. DOI: 10.1186/s40168-015-0070-0.

19. Kostic $A D$, Xavier RJ, Gevers $D$. The microbiome in inflammatory bowel disease: current status and the future ahead. Gastroenterology 2014;146(6):1489-1499. DOI: 10.1053/j.gastro.2014.02.009.

20. Moayyedi P, Surette MG, Kim PT, et al. Fecal microbiota transplantation induces remission in patients with active ulcerative colitis in a randomized controlled trial. Gastroenterology 2015;149(1):102-109. e6. DOI: 10.1053/j.gastro.2015.04.001.

21. Paramsothy S, Kamm MA, Kaakoush NO, et al. Multidonor intensive Fecal microbiota transplantation for active ulcerative colitis: a randomised placebo-controlled trial. Lancet 2017;389(10075): 1218-1228. DOI: 10.1016/S0140-6736(17)30182-4.

22. Rossen NG, Fuentes S, van der Spek MJ, et al. Findings from a randomized controlled trial of fecal transplantation for patients with ulcerative colitis. Gastroenterology 2015;149(1):110-118.e4. DOI: 10.1053/j.gastro.2015.03.045.

23. Costello SP, Hughes PA, Waters $\mathrm{O}$, et al. Effect of fecal microbiota transplantation on 8-week remission in patients with ulcerative colitis: a randomized clinical trial. JAMA 2019;321(2):156-164. DOI: 10.1001/jama.2018.20046.

24. Wang L, Alammar N, Singh R, et al. Gut microbial dysbiosis in the irritable bowel syndrome: a systematic review and meta-analysis of case-control studies. J Acad Nutr Diet 2020;120(4):565-586. DOI: 10.1016/j.jand.2019.05.015. 
25. Ng KM, Ferreyra JA, Higginbottom SK, et al. Microbiotaliberated host sugars facilitate post-antibiotic expansion of enteric pathogens. Nature 2013;502(7469):96-99. DOI: 10.1038/ nature12503.

26. Chen HC, Chang CC, Mau WJ, et al. Evaluation of $\mathrm{N}$ acetylchitooligosaccharides as the main carbon sources for the growth of intestinal bacteria. FEMS Microbiol Lett 2002;209(1):53-56. DOI: 10.1111/j.1574-6968.2002.tb11108.x.

27. Ellermann M, Arthur JC. Siderophore-mediated iron acquisition and modulation of host-bacterial interactions. Free Radic Biol Med 2017;105:68-78. DOI: 10.1016/j.freeradbiomed.2016.10.489.

28. Kopke M, Straub M, Durre P. Clostridium difficile is an autotrophic bacterial pathogen. PLoS One. 2013;8(4):e62157. DOI: 10.1371/journal. pone.0062157.

29. Hofmann AF, Hagey LR. Bile acids: chemistry, pathochemistry, biology, pathobiology, and therapeutics. Cell Mol Life Sci 2008;65(16): 2461-2483. DOI: 10.1007/s00018-008-7568-6.

30. Weingarden AR, Chen C, Bobr A, et al. Microbiota transplantation restores normal fecal bile acid composition in recurrent Clostridium difficile infection. Am J Physiol Gastrointest Liver Physiol 2014;306(4):G310-G319. DOI:10.1152/ajpgi.00282.2013.

31. Hooper LV, Macpherson AJ. Immune adaptations that maintain homeostasis with the intestinal microbiota. Nat Rev Immunol 2010;10(3):159-169. DOI: 10.1038/nri2710.

32. Francino MP. Early development of the gut microbiota and immune health. Pathogens 2014;3(3):769-790. DOI: 10.3390/ pathogens3030769.

33. Mazmanian SK, Round JL, Kasper DL. A microbial symbiosis factor prevents intestinal inflammatory disease. Nature 2008;453(7195): 620-625. DOI: 10.1038/nature07008.

34. Glover LE, Lee JS, Colgan SP. Oxygen metabolism and barrier regulation in the intestinal mucosa. J Clin Invest 2016;126(10): 3680-3688. DOI: 10.1172/JCI84429.

35. Burrello C, Garavaglia F, Cribiù FM, et al. Therapeutic Fecal microbiota transplantation controls intestinal inflammation through IL10 secretion by immune cells. Nat Commun 2018;9(1):5184. DOI: 10.1038/ s41467-018-07359-8.

36. Ekmekciu I, Von Klitzing E, Fiebiger $U$, et al. Immune responses to broad-spectrum antibiotic treatment and fecal microbiota transplantation in mice. Front Immunol 2017;8:397. DOI: 10.3389/ fimmu.2017.00397.

37. Ekmekciu I, Von Klitzing E, Neumann C, et al. Fecal microbiota transplantation, commensal Escherichia coli and Lactobacillus johnsonii strains differentially restore intestinal and systemic adaptive immune cell populations following broad-spectrum antibiotic treatment. Front Microbiol 2017;8:2430. DOI: 10.3389/ fmicb.2017.02430.

38. van Nood E, Vrieze A, Nieuwdorp M, et al. Duodenal infusion of donor feces for recurrent Clostridium difficile. N Engl J Med 2013;368(5): 407-415. DOI: 10.1056/NEJMoa1205037.

39. Cammarota G, Masucci L, laniro G, et al. Randomized clinical trial: fecal microbiota transplantation by colonoscopy vs. vancomycin for the treatment of recurrent Clostridium difficile infection. Aliment Pharmacol Ther 2015;41(9):835-843. DOI: 10.1111/apt.13144.

40. Kelly CR, Khoruts A, Staley C, et al. Effect of fecal microbiota transplantation on recurrence in multiply recurrent clostridium difficile infection: a randomized trial. Ann Intern Med 2016;165(9): 609-616. DOI: 10.7326/M16-0271.

41. Vaishnavi C. Fecal microbiota transplantation for management of Clostridium difficile infection. Indian J Gastroenterol 2014;33(4): 301-307. DOI: 10.1007/s12664-014-0459-x.

42. Quraishi MN, Widlak M, Bhala N, et al. Systematic review with meta-analysis: the efficacy of faecal microbiota transplantation for the treatment of recurrent and refractory Clostridium difficile infection. Aliment Pharmacol Ther 2017;46(5):479-493. DOI: 10.1111/ apt.14201.

43. Hui W, Li T, Liu W, et al. Fecal microbiota transplantation for treatment of recurrent $C$. difficile infection: An updated randomized controlled trial meta-analysis. PLoS One 2019;14(1):e0210016. DOI: 10.1371/ journal.pone.0210016.

44. Hvas CL, Dahl Jørgensen SM, Jørgensen SP, et al. Fecal microbiota transplantation is superior to fidaxomicin for treatment of recurrent clostridium difficile infection. Gastroenterology 2019;156(5): 1324-1332. DOI: 10.1053/j.gastro.2018.12.019.

45. Fischer M, Sipe BW, Rogers NA, et al. Fecal microbiota transplantation plus selected use of vancomycin for severe-complicated Clostridium difficile infection: description of a protocol with high success rate. Aliment Pharmacol Ther 2015;42(4):470-476. DOI: 10.1111/ apt.13290.

46. Fischer M, Sipe B, Cheng YW, et al. Fecal microbiota transplant in severe and severe-complicated Clostridium difficile: a promising treatment approach. Gut Microbes 2017;8(3):289-302. DOI: 10.1080/19490976.2016.1273998.

47. Ianiro G, Masucci L, Quaranta G, et al. Randomized clinical trial: Fecal microbiota transplantation by colonoscopy plus vancomycin for the treatment of severe refractory Clostridium difficile infection-single versus multiple infusions. Aliment Pharmacol Ther 2018;48(2):152-159. DOI: 10.1111/apt.14816.

48. Hocquart M, Lagier JC, Cassir N, et al. Early fecal microbiota transplantation improves survival in severe Clostridium difficile infections. Clin Infect Dis 2018;66(5):645-650. DOI: 10.1093/cid/ cix762.

49. Juul FE, Garborg K, Bretthauer M, et al. Fecal microbiota transplantation for primary clostridium difficile infection. $N$ Engl J Med 2018;378(26):2535-2536. DOI: 10.1056/NEJMc1803103.

50. Camacho-Ortiz A, Gutiérrez-Delgado EM, Garcia-Mazcorro JF, et al. Randomized clinical trial to evaluate the effect of fecal microbiota transplant for initial Clostridium difficile infection in intestinal microbiome. PLoS One 2017;12(12):e0189768. DOI: 10.1371/journal. pone. 0189768 .

51. Cammarota G, laniro G, Tilg H, et al. European FMT Working Group. European consensus conference on faecal microbiota transplantation in clinical practice. Gut 2017;66(4):569-580. DOI: 10.1136/gutjnl-2016313017.

52. Bennet JD, Brinkman M. Treatment of ulcerative colitis by implantation of normal colonic flora. Lancet 1989;1(8630):164. DOI: 10.1016/s01406736(89)91183-5.

53. Borody TJ, George L, Andrews $\mathrm{P}$, et al. Bowel-flora alteration: a potential cure for inflammatory bowel disease and irritable bowel syndrome? Med J Aust 1989;150(10):604.

54. Butta H, Kapoor A, Sibal A, et al. Fecal microbial transplant: a new hope of treatment for ulcerative colitis. Int J Med Public Health 2018;8(1):7-10. DOI: 10.5530/ijmedph.2018.1.2.

55. Seth AK, Rawal P, Bagga R, et al. Successful colonoscopic fecal microbiota transplantation for active ulcerative colitis: first report from India. Indian J Gastroenterol 2016;35(5):393-395. DOI: 10.1007/ s12664-016-0696-2.

56. Goyal A, Yeh A, Bush BR, et al. Safety, clinical response, and microbiome findings following fecal microbiota transplant in children with inflammatory bowel disease. Inflamm Bowel Dis 2018;24(2): 410-421. DOI: 10.1093/ibd/izx035.

57. Sood A, Mahajan R, Juyal G, et al. Efficacy of fecal microbiota therapy in steroid dependent ulcerative colitis: a real world intentionto-treat analysis. Intest Res 2019;17(1):78-86. DOI: 10.5217/ ir.2018.00089.

58. Moayyedi P. Update on fecal microbiota transplantation in patients with inflammatory bowel disease. Gastroenterol Hepatol 2018;14(5):319-322.

59. Sood A, Mahajan R, Singh A, et al. Role of fecal microbiota transplantation for maintenance of remission in patients with ulcerative colitis: a pilot study. J Crohns Colitis 2019;13(10):1311-1317. DOI: $10.1093 /$ ecco-jcc/jjz060.

60. Li Q, Zhang T, Ding X, et al. Enhancing patient adherence to fecal microbiota transplantation maintains the long-term clinical effects in ulcerative colitis. European J Gastroenterol Hepatol 2020;32(8): 955-962. DOI: 10.1097/MEG.0000000000001725. 
61. Sokol H, Landman C, Seksik P, et al. Fecal microbiota transplantation to maintain remission in Crohn's disease: a pilot randomized controlled study. Microbiome 2020;8(1):12. DOI: 10.1186/s40168020-0792-5.

62. Yang Z, Bu C, Yuan W, et al. Fecal microbiota transplant via endoscopic delivering through small intestine and colon: no difference for Crohn's disease. Dig Dis Sci 2020;65(1):150-157. DOI: 10.1007/s10620-01905751-y.

63. Vaughn BP, Vatanen $T$, Allegretti JR, et al. Increased intestinal microbial diversity following fecal microbiota transplant for active Crohn's disease. Inflamm Bowel Dis 2016;22(9):2182-2190. DOI: $10.1097 / \mathrm{MIB} .0000000000000893$.

64. Wang H, Cui B, Li Q, et al. The safety of fecal microbiota transplantation for Crohn's disease: findings from a long-term study. Adv Ther 2018;35(11):1935-1944. DOI: 10.1007/s12325-018-0800-3.

65. Carroll IM, Ringel-Kulka T, Siddle JP, et al. Alterations in composition and diversity of the intestinal microbiota in patients with diarrheapredominant irritable bowel syndrome. Neurogastroenterol Motil 2012;24(6):521-530, e248.DOI: 10.1111/j.1365-2982.2012. 01891.x.

66. Tap J, Derrien $\mathrm{M}$, Törnblom $\mathrm{H}$, et al. Identification of an intestinal microbiota signature associated with severity of irritable bowel syndrome. Gastroenterology 2017;152(1):111-123.e8. DOI: 10.1053/j. gastro.2016.09.049.

67. Shukla R, Ghoshal U, Dhole TN, et al. Fecal microbiota in patients with irritable bowel syndrome compared with healthy controls using realtime polymerase chain reaction: an evidence of dysbiosis. Dig Dis Sci 2015;60(10):2953-2962. DOI: 10.1007/s10620-015-3607-y.

68. Halkjær SI, Christensen AH, Lo BZ, et al. Fecal microbiota transplantation alters gut microbiota in patients with irritable bowel syndrome: results from a randomized, double-blind placebo-controlled study. Gut 2018;67(12):2107-2115. DOI: 10.1136/gutjnl-2018-316434.

69. Johnsen PH, Hilpüsch F, Cavanagh JP, et al. Fecal microbiota transplantation versus placebo for moderate-to-severe irritable bowel syndrome: a double-blind, randomized, placebo-controlled, parallel-group, single-center trial. Lancet Gastroenterol Hepatol 2018;3(1):17-24. DOI: 10.1016/S2468-1253(17)30338-2.

70. Holvoet T, Joossens $M$, Jerina B, et al. Fecal microbiota transplantation in irritable bowel syndrome with predominant abdominal bloating: results from a double blind, placebo-controlled clinical trial. Gastroenterology 2018;154(6):S-130. DOI: 10.1016/S00165085(18)30860-6.

71. El-Salhy M, Hatlebakk JG, Gilja OH, et al. Efficacy of faecal microbiota transplantation for patients with irritable bowel syndrome in a randomized, double-blind, placebo-controlled study. Gut. 2020;69(5):859-867. DOI: 10.1136/gutjnl-2019-319630.

72. Lahtinen $P$, Jalanka J, Hartikainen A, et al. Randomized clinical trial: Fecal microbiota transplantation versus autologous placebo administered via colonoscopy in irritable bowel syndrome. Aliment Pharmacol Ther 2020;51(12):1321-1331. DOI: 10.1111/apt.15740.

73. Ianiro G, Eusebi LH, Black CJ, et al. Systematic review with metaanalysis: efficacy of Fecal microbiota transplantation for the treatment of irritable bowel syndrome. Aliment Pharmacol Ther 2019;50(3):240-248. DOI: 10.1111/apt.15330.

74. Xu D, Chen VL, Steiner CA, et al. Efficacy of fecal microbiota transplantation in irritable bowel syndrome: a systematic review and meta-analysis. Am J Gastroenterol 2019;114(7):1043-1050. DOI: 10.14309/ajg.0000000000000198.

75. Sood A, Ahuja V, Kedia S, et al. Diet and inflammatory bowel disease: The Asian Working Group guidelines [published correction appears in Indian J Gastroenterol 2019;38(6):558-559]. Indian J Gastroenterol 2019;38(3):220-246. DOI: 10.1007/s12664-019-00976-1.

76. Wilson BC, Vatanen T, Cutfield WS, et al. The super-donor phenomenon in fecal microbiota transplantation. Front Cell Infect Microbiol 2019;9:2. DOI: 10.3389/fcimb.2019.00002.

77. Zhang $\mathrm{F}$, Zhang $\mathrm{T}$, Zhu $\mathrm{H}$, et al. Evolution of fecal microbiota transplantation in methodology and ethical issues. Curr Opin Pharmacol 2019;49:11-16. DOI: 10.1016/j.coph.2019.04.004.
78. Lee $\mathrm{CH}$, Steiner T, Petrof EO, et al. Frozen vs fresh fecal microbiota transplantation and clinical resolution of diarrhea in patients with recurrent Clostridium difficile infection: a randomized clinical trial. JAMA 2016;315(2):142-149. DOI: 10.1001/jama.2015. 18098.

79. Papanicolas $L E$, Choo JM, Wang Y, et al. Bacterial viability in fecal transplants: which bacteria survive? EBioMedicine 2019;41:509-516. DOI: 10.1016/j.ebiom.2019.02.023.

80. Jiang ZD, Alexander A, Ke S, et al. Stability and efficacy of frozen and lyophilized Fecal microbiota transplant (FMT) product in a mouse model of Clostridium difficile infection (CDI). Anaerobe 2017;48:110-114. DOI: 10.1016/j.anaerobe.2017.08.003.

81. Jiang ZD, Ajami NJ, Petrosino JF, et al. Randomized clinical trial: faecal microbiota transplantation for recurrent Clostridium difficile infection - fresh, or frozen, or lyophilised microbiota from a small pool of healthy donors delivered by colonoscopy. Aliment Pharmacol Ther 2017;45(7):899-908. DOI: 10.1111/apt.13969.

82. Costello SP, Tucker EC, La Brooy J, et al. Establishing a fecal microbiota transplant service for the treatment of Clostridium difficile infection. Clin Infect Dis 2016;62(7):908-914. DOI: 10.1093/cid/ civ994.

83. Keshteli AH, Millan B, Madsen KL. Pretreatment with antibiotics may enhance the efficacy of Fecal microbiota transplantation in ulcerative colitis: a meta-analysis. Mucosal Immunol 2017;10(2):565-566. DOI: 10.1038/mi.2016.123.

84. Freitag $\mathrm{TL}$, Hartikainen $\mathrm{A}$, Jouhten $\mathrm{H}$, et al. Minor effect of antibiotic pre-treatment on the engraftment of donor microbiota in fecal transplantation in mice. Front Microbiol 2019;10:2685. DOI: 10.3389/ fmicb.2019.02685.

85. Peng Z, Xiang J, He Z, et al. Colonic transendoscopic enteral tubing: a novel way of transplanting fecal microbiota. Endosc Int Open 2016;4(6):E610-E613. DOI: 10.1055/s-0042-105205.

86. Gweon TG, Kim J, Lim CH, et al. Fecal microbiota transplantation using upper gastrointestinal tract for the treatment of refractory or severe complicated clostridium difficile infection in elderly patients in poor medical condition: the first study in an Asian country. Gastroenterol Res Pract 2016;2016:2687605. DOI: 10.1155/2016/2687605.

87. Wang $S$, Xu M, Wang W, et al. Systematic review: adverse events of fecal microbiota transplantation. PLoS One 2016;11(8):e0161174. DOI: 10.1371/journal.pone.0161174.

88. Kao D, Roach B, Silva M, et al. Effect of oral capsule- vs colonoscopydelivered fecal microbiota transplantation on recurrent Clostridium difficile infection: a randomized clinical trial. JAMA 2017;318(20): 1985-1993. DOI: 10.1001/jama.2017.17077.

89. Steube A, Vital M, Grunert P, et al. Long-term multidonor fecal microbiota transfer by oral capsules for active ulcerative colitis. J Crohns Colitis 2019;13(11):1480-1481. DOI: 10.1093/ecco-jcc/ jjz073.

90. Mizuno S, Nanki K, Matsuoka K, et al. Single fecal microbiota transplantation failed to change intestinal microbiota and had limited effectiveness against ulcerative colitis in Japanese patients. Intest Res 2017;15(1):68-74. DOI: 10.5217/ir.2017.15.1.68.

91. Staley C, Kaiser T, Vaughn BP, et al. Durable long-term bacterial engraftment following encapsulated fecal microbiota transplantation to treat Clostridium difficile infection. mBio 2019;10(4). DOI: 10.1128/ mBio.01586-19.

92. Tabbaa OM, Aboelsoud MM, Mattar MC. Long-term safety and efficacy of fecal microbiota transplantation in the treatment of Clostridium difficile infection in patients with and without inflammatory bowel disease: a tertiary care centre's experience. Gastroenterology Res 2018;11(6):397-403. DOI: 10.14740/gr1091.

93. Lee $\mathrm{CH}$, Chai J, Hammond $\mathrm{K}$, et al. Long-term durability and safety of Fecal microbiota transplantation for recurrent or refractory Clostridioides difficile infection with or without antibiotic exposure. Eur J Clin Microbiol Infect Dis 2019;38(9):1731-1735. DOI: 10.1007/ s10096-019-03602-2.

94. Jalanka J, Hillamaa A, Satokari R, et al. The long-term effects of fecal microbiota transplantation for gastrointestinal symptoms and general 
health in patients with recurrent Clostridium difficile infection. Aliment Pharmacol Ther 2018;47(3):371-379. DOI: 10.1111/apt.14443.

95. Sood A, Singh A, Mahajan R, et al. Acceptability, tolerability, and safety of fecal microbiota transplantation in patients with active ulcerative colitis (AT\&S Study). J Gastroenterol Hepatol 2020;35(3):418-424. DOI: 10.1111/jgh.14829.
96. DeFilipp Z, Bloom PP, Torres Soto M, et al. Drug-Resistant E. coli bacteremia transmitted by fecal microbiota transplant. N Engl J Med 2019;381(21):2043-2050. DOI: 10.1056/NEJMoa1910437.

97. Cotton PB, Eisen GM, Aabakken L, et al. A lexicon for endoscopic adverse events: report of an ASGE workshop. Gastrointest Endosc 2010;71(3):446-454. DOI: 10.1016/j.gie.2009.10.027. 